\title{
Magnetic resonance lineshapes in powdered and amorphous systems
}

\author{
P RAGHUNATHAN* and S C SIVASUBRAMANIAN \\ Department of Chemistry, Indian Institute of Technology, Kanpur 208016, India
}

\begin{abstract}
A survey is made of the theory and applications of EPR and NMR absorption lineshapes obseryed in powdered and amorphous materials. The 'spin Hamiltonian' and 'resonance condition' formalisms are reviewed, and EPR and NMR lineshapes are discussed which typify the singularity characteristics in powdered materials. In the amorphous or 'glassy' state, the measurable spin resonance parameters often have to be viewed as being 'randomly distributed' according to a probability density function. Several recent probability-theory based approaches to lineshape computation for modelling the amorphous state are discussed.
\end{abstract}

Keywords. EPR; NMR; lineshapes; powder; amorphous materials.

\section{Introduction}

Over the past several decades, amorphous solids have continued to be of tremendous interest to chemists, physicists and material scientists alike, and the question of 'how to recover the solid state physics without the periodic lattice' (Müller-Warmuth and Eckert 1982) has challenged theoreticians and experimentalists alike. Among magnetic resonance spectroscopists, the interpretation of a variety of resonance absorption 'patterns' of amorphous systems, as well as the extraction of magnetic interaction parameters therefrom, often pose a fascinating problem. This is because several of the important molecular tensor properties such as the electron paramagnetic resonance (EPR) $g$-factors, hyperfine $(A)$ and zero-field $(D)$ interactions, the nuclear quadrupole interaction $(Q)$ etc., appear distributed and spectroscopic information has then to be recovered by modelling the paramagnetic system by means of detailed lineshape simulation procedures.

The purpose of this survey is to review briefly the 'spin Hamiltonian' formalism representing the various interaction parameters and then to show how resonance conditions based on these spin Hamiltonians lead to typical and characteristic singularities in the absorption lineshapes of powdered materials. The formalism will then be modified in a variety of ways for the analysis of the 'glassy' or amorphous state. Finally we shall consider, with representative examples, the concepts and interpretive guidelines that have been demonstrated in recent years for the analysis of EPR and NMR lineshapes in typical amorphous and glass structures.

\section{The spin Hamiltonian and the resonance condition}

In the laboratory, one customarily records the magnetic resonance spectrum of a solid sample as the first derivative of resonant absorption versus the applied magnetic field,

* To whom all correspondence should be addressed. 
H. From this spectral 'shape' one then derives the appropriate 'spin Hamiltonian', which is nothing but an expression in matrix representation for the interaction energy of the paramagnetic spin vector (nuclear spin $I$ in the case of NMR and electron spin $S$ in the case of EPR) with the field vector, $H$. The various parameters of the spin Hamiltonian are then related back to the chemical bonding of the environment by means of appropriate theories of molecular electronic structure.

The aforementioned spin Hamiltonian denoted in its most general form by $\mathscr{H}$, is phenomenologically set up such that it has a leading term representing the 'Zeeman' interaction between $\mathbf{H}$ and the $\mathbf{S}$ or $\mathbf{I}$ spin vector, followed by successively smaller 'perturbations' representing various tensorial couplings. In EPR, it takes the most general form

$$
\mathscr{H}_{\mathrm{EPR}}=\boldsymbol{\beta}_{\mathrm{e}} \mathbf{H} \cdot \overrightarrow{\mathbf{g}} \cdot \mathbf{S}+\mathbf{I} \cdot \overrightarrow{\mathbf{A}} \cdot \mathbf{S}+\mathbf{S} \cdot \overrightarrow{\mathbf{D}} \cdot \mathbf{S}+\mathbf{I} \cdot \overrightarrow{\mathbf{Q}} \cdot \mathbf{I}-\gamma \hbar \mathbf{I} \cdot \mathbf{H},
$$

where the leading (or zero-order) Zeeman term defines the molecular $g$-tensor. The second term is the electron spin-nuclear spin 'hyperfine' interaction $(A)$, and the third term (operative in the case of several unpaired electron spins being present in the valence orbital of the paramagnetic centre, e.g., $\mathrm{Mn}^{2+}$ ) is the 'fine structure' (or crystal field) interaction $(D)$. The last two terms of (1) are the nuclear quadrupolar $(Q)$ and the nuclear Zeeman interaction, respectively, and are usually very small members in the above 'hierarchy' of perturbations (Raghunathan 1981), and could become important in special instances as we shall exemplify later on. In (1), $\beta_{e}$ is the fundamental "electronic Bohr magneton' unit, and $\gamma$ is another fundamental interaction constant called the nuclear magnetogyric ratio. $\overrightarrow{\mathbf{g}}, \overrightarrow{\mathbf{A}}, \overrightarrow{\mathbf{D}}$ and $\overrightarrow{\mathbf{Q}}$ are symmetric second-rank tensors representing the anisotropic nature of the respective couplings.

In an entirely analogous fashion, one may set up an appropriate NMR spin Hamiltonian. Foremost among the interactions contributing to resonance lineshapes are the internuclear magnetic dipole-dipole interactions and (for nuclei with $I>\frac{1}{2}$ ) the nuclear electric quadrupole interaction with the surrounding electric field gradient. In many cases, one or the other of these two interactions can be neglected, e.g., for protons and ${ }^{19} \mathrm{~F}$ the quadrupolar interaction is zero, whereas for deuterons, ${ }^{11} \mathrm{~B},{ }^{14} \mathrm{~N}$ etc., the quadrupolar interaction dominates. On this basis the solids of interest in NMR spectroscopy are usually referred to as dipolar and quadrupolar. Having established this dichotomy, we shall here consider the quadrupolar (amorphous) solids only, since dipolar solid lineshapes have been reviewed in detail in several places (see, e.g., Raghunathan 1982).

We shall, accordingly, write the NMR spin Hamiltonian as

$$
\begin{aligned}
\mathscr{H}_{\mathrm{NMR}} & =-\gamma \hbar \mathbf{I} \cdot \mathbf{H}+\mathbf{I} \cdot \overrightarrow{\mathbf{Q}} \cdot \mathbf{I} \\
& =H_{0}+H_{Q} .
\end{aligned}
$$

In the above, the second term written in terms of appropriate components of the nuclear spin becomes

$$
H_{Q}=\frac{e^{2} q Q}{4 I(2 I-1) \hbar}\left[3 I_{z}^{2}-\mathbf{I}^{2}+\eta\left(\mathbf{I}_{+}^{2}-\mathbf{I}_{-}^{2}\right)\right],
$$

where $e^{2} q Q$ is the experimentally measurable 'quadrupolar coupling constant' parameter involving the electric quadrupole moment, $e Q$, of the nucleus, and a component $q$ of the electric field gradient (EFG) tensor with principal axis components 
$q_{x x}, q_{y y}$ and $q_{z z}(=q) . \eta$ is called the 'asymmetry parameter', and is given by

$$
\eta=\frac{q_{x x}-q_{y y}}{q_{z z}} \text { where }\left|q_{x x}\right| \leqslant\left|q_{y y}\right| \leqslant\left|q_{z z}\right| \text {. }
$$

When $H_{Q}<H_{0}$, we could consider the former as a perturbing term which splits the magnetic resonance absorption line into $2 I+1$ components.

Relating the experimental spectra to (1) and (2) requires that the spin Hamiltonian be diagonalised to yield the 'resonance condition', $H_{m}$-an expression for the magnetic field at which resonance occurs as a function of the principal-axis values of the tensor quantities, the resonance frequency $v$, and the magnetic quantum number $m_{S}$ or $m_{I}$. Of course, $\mathbf{H}$, being a vector, is specified both by its magnitude and its orientation with respect to the principal axis coordinate systems of the interaction tensor. The orientation of $\mathbf{H}$ with respect to the tensor axes in (1) and (2) will be specified by the Euler angles $\theta$ and $\phi$, as defined in figure 1. The EPR resonance condition for the $\left(m_{S}-1\right)$ $\rightarrow m_{S}, m_{l}=0$ transitions, to first-order, is given by (Taylor et al 1975):

$$
h v=g \beta_{e} H+(A m / g)
$$

where

$$
\begin{aligned}
& g=\left[g_{1}^{2} \sin ^{2} \theta \sin ^{2} \phi+g_{2}^{2} \sin ^{2} \theta \cos ^{2} \phi+g_{3}^{2} \cos ^{2} \theta\right]^{1 / 2}, \\
& A=\left[A_{1}^{2} g_{1}^{2} \sin ^{2} \theta \sin ^{2} \phi+A_{2}^{2} g_{2}^{2} \sin ^{2} \theta \cos ^{2} \phi+A_{3}^{2} g_{3}^{2} \cos ^{2} \theta\right]^{1 / 2} .
\end{aligned}
$$

The resonance conditions based on the NMR Hamiltonian (2) may be established in a manner analogous to the above by recognising that, now, $\theta$ and $\phi$ are the Euler angles relating the direction of the magnetic field $\mathbf{H}$ and the symmetry axes of the EFG tensor. When perturbation theory is considered only upto first order (Cohen and Reif 1957),

$$
v_{m}^{(1)}=\frac{E_{m}^{(1)}-1-E_{m}^{(1)}}{\hbar}=\frac{1}{2} v_{0}\left(m-\frac{1}{2}\right)\left(3 \cos ^{2} \theta-1\right)
$$

where $v_{0}$ is the central resonance frequency of the nucleus in the absence of quadrupolar effects. We see from (5) that the first-order shift $v_{m}^{(1)}$ vanishes for $m=\frac{1}{2}$. For half-integral spins, the central transition $\frac{1}{2} \leftrightarrow-\frac{1}{2}$ is therefore not shifted in first order by quadrupolar interaction. The frequencies of the other allowed lines are, however, shifted and satellite

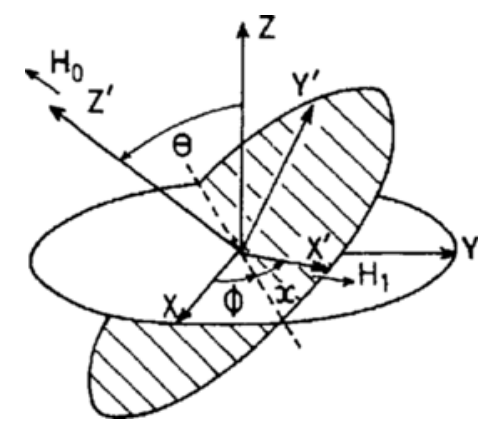

Figure 1. A general orientation of the magnetic field with respect to the molecular tensor axes, $X Y Z$ represents the molecule-centred principal axis system; $X^{\prime}, Y^{\prime}, Z^{\prime}$ are the laboratory reference axes with the field $H$ along $Z$ ' and oscillating field $H_{1}$ along $X$ '. The Euler angles are also marked. 
lines corresponding to transitions $m \leftrightarrow m-1$ appear symmetrically on either side of the central line.

For the NMR of quadrupolar solids, therefore, perturbation theory considered upto second order is more revealing, and we obtain the second-order shifted resonance line, $v_{m}^{(2)}$, from the expression of $E_{m}^{(1)}$ which is an odd function of $m$ (Jones et al 1963). In particular, when the EFG has axial symmetry $(\eta=0)$, the central $m_{I}=+\frac{1}{2} \leftrightarrow m_{I}=-\frac{1}{2}$ transition becomes shifted according to

$$
v_{1 / 2}^{(2)}=(3 / 64)\left(1 / v_{0}\right)\left(e^{2} q Q\right)^{2}\left(1-9 \cos ^{2} \theta\right)\left(1-\cos ^{2} \theta\right) .
$$

\section{Powder lineshapes and shape functions}

In a finely crushed 'powder' specimen, spin sites occur with all possible orientations of their axes with respect to the direction of $\mathbf{H}$. We consider these orientations as lying along the radii of a sphere, and partition the surface of the sphere into a grid determined by equal intervals of $\theta$ and $\phi$. As can be readily seen, the surface area, and hence the solid angle, depends on the angle $\theta$ (see figure 2). In spherical polar coordinates, the differential element of solid angle $d \Omega=\sin \theta \mathrm{d} \theta \mathrm{d} \phi$. (This leads to the interesting result that there will be fewer spin sites within a given angular range near the parallel $\left(\theta=0^{\circ}\right)$ than within the same angular range near the perpendicular orientation $\left(\theta=90^{\circ}\right)$; therefore, for a near-axial system, the perpendicular features of the spectrum will be more intense than the 'parallel' ones.)

We can now, for any given transition, calculate a representative intensity for each area. Multiplying this intensity by the area and summing over all such areas should give, as the number of areas becomes large, the integrated intensity of the total powder 'lineshape'. Since, of course, the intensity of a transition varies with the value of the magnetic field which is being swept in the experiment, this process would have to be iterated for each chosen value of $H$ to obtain the "density of resonance

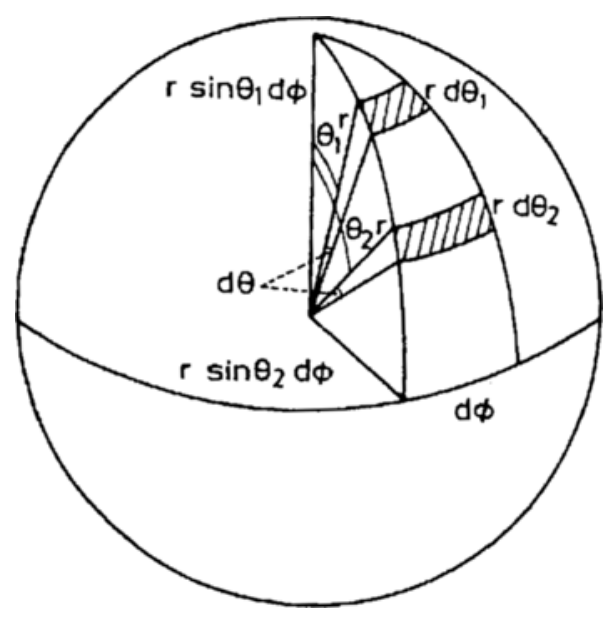

Figure 2. Dependence of the solid angle (area) on $\theta$. Since the area changes as $\sin \theta$, the number of orientations in a given volume element arising from a grid determined by equal $\theta$ and equal $\phi$ intervals goes as $\sin \theta$. 
fields'. Since we are interested only in relative intensities, we need to multiply the intensity of each area only by $\sin \theta$ if both $\theta$ as well as $\phi$ are stepped up in equal increments in our calculation. If we use equal increments of $\cos \theta$, we do not need any multiplier since $\mathrm{d}(\cos \theta)=-\sin \theta \mathrm{d} \theta$.

We thus make the important generalization that the magnetic 'resonance condition' for powders may be thought of as a surface in $(\cos \theta, \phi)$ space and can be written formally as $H_{m}=H_{m}(\cos \theta, \phi)$ with $m$ denoting the appropriate transition, e.g., the $m_{I} \rightarrow\left(m_{I}-1\right)$ NMR transition or the $\left(m_{S-1}\right) \rightarrow m_{S}$ EPR transition. Accordingly in all numerical techniques of lineshape analysis (Maruani et al 1968; Taylor et al 1968; McDowell et al 1973; Jinguji et al 1976), the resonance condition is solved for all orientations given by a fine grid in $\cos \theta-\phi$ space. An equivalent, and often faster, computational procedure is to solve for the densities of resonance fields at a random distribution of points in $\cos \theta-\phi$ space generated by a Monte Carlo routine, and this is the procedure of choice in our laboratory.

By using either procedure, we see that the differential probability, $\mathrm{d} P(H)$, of the resonance condition for a paramagnetic powder 'site' lying between $H$ and $H+\mathrm{d} H$ may be written in the normalised form (Jinguji et al 1976),

$$
\begin{aligned}
\mathrm{d} P(H) & =\mathrm{d} \Omega(\theta, \phi) / 4 \pi \\
& =\mathrm{d} \cos \theta \mathrm{d} \phi / 4 \pi,
\end{aligned}
$$

and the spectral lineshape at each resonance field obtained as

$$
\frac{\mathrm{d} P(H)}{\mathrm{d} H}=(4 \pi)^{-1} \frac{\mathrm{d} \Omega}{\mathrm{d} H}=(4 \pi)^{-1}\left(\frac{\mathrm{d} H}{\mathrm{~d} \Omega}\right)^{-1} .
$$

The overall 'shape function' $S(H)$ which is of interest to us is therefore the weighted distribution of the resonance condition over all equally probable elements of $d \Omega$ integrated over all the allowed transitions. The lineshape information is stored in the inherent anisotropy of the spin site which is the same for all crystallites of the powder specimen. The Euler angles and the random orientations of the crystallites are the weighting factors which spread this information between the largest and smallest values in the 'distribution of resonance fields'. One writes $S(H)$ as

$$
S(H)=\int_{0}^{2 \pi} \mathrm{d} \phi \int_{0}^{\pi} \sin \theta \mathrm{d} \theta\left(H_{m}\right) P_{m}(\theta, \phi),
$$

where $P_{m}(\theta, \phi)$ is the transition probability for the $m$ th component of the spectral absorption, being proportional to $S(S+1)-m_{S}\left(m_{S}-1\right)$ in the case of EPR and $I(I+1)-m_{I}\left(m_{I}-1\right)$ in the case of NMR. The $P_{m}(\theta, \phi)$ factor is usually independent of the solid angle and may therefore be taken outside the integral, although special cases of angular dependence of $P_{m}(\theta, \phi)$ have been discussed in the literature from time to time (Bleaney 1960; Isomoto et al 1970).

It is seen from (8) that lineshape singularities arise automatically at field values corresponding to critical points (Kottis and Lefebvre 1964; Maruani et al 1968; Coope 1969) of the function $H_{m}=H(\cos \theta, \phi)$, i.e., points where

$$
\left(\frac{\partial H}{\partial \cos \theta}\right)_{x, y}=\left(\frac{\partial H}{\partial \phi}\right)_{x, y}=0
$$


$x$ and $y$ being the coordinates of the critical point. A saddle point in the $H(\cos \theta, \phi)$ surface at location $(x, y)$ indicates the presence of a divergence in the powder pattern at the field $H=H(x, y)$, whereas a relative extremum will give rise to a shoulder or step at $H(x, y)$. However, dipolar interaction mechanisms between neighbouring groups of spins somewhat broaden and smear out these singularities and therefore, in most actual cases, smoothed out shoulders and divergences are seen in the powder pattern at fields corresponding to $\mathbf{H}$ being aligned along one of the principal axes of the interaction tensor. For diagnostic purposes it is often sufficient to determine the locations of these principal features in the first and second derivative powder patterns (Weil and Hecht 1963; Lee 1981; Lee et al 1985).

On the other hand, to enable the entire theoretical 'step-shoulder-divergence' lineshapes to fit the experimental spectra, the ensemble of line positions calculated according to (9) is often convoluted with a suitable broadening function appropriate to the type of amorphous solid under study. For example, for the most often used Gaussian-shaped line of full width at half height $w$ which characterises the small dipolar broadening mechanisms in most solids, the broadening function may be written as

$$
f\left(H_{m}-H\right) \propto w^{-1} \exp \left[-4 \ln 2\left(\left(H_{m}-H\right) / w\right)^{2}\right] .
$$

A second source of line-broadening may be brought about by exchange interaction mechanisms in some solids (Bloch 1946; Van Vleck 1948), which may be approximated by the Lorentzian broadening function,

$$
f\left(H_{m}-H\right) \propto w\left[\left(w^{2} / 4\right)+\left(H_{m}-H\right)^{2}\right]^{-1},
$$

where $w$ is the full width at half-height of the broadened line. In a general sense one may therefore consider the final, convoluted form of $S(H),(9)$, as

$$
S(H)=\int_{0}^{2 \pi} \mathrm{d} \phi \int_{0}^{\pi} \sin \theta \mathrm{d} \theta f\left(H_{m}-H\right) P_{m}(\theta, \phi) .
$$

This is the standard lineshape function used whenever the powder sample may be thought of as a randomly oriented ensemble of otherwise identical sites. Although examples of these lineshapes abound in the literature, here we shall briefly typify the most frequently observed classes of EPR lineshape singularities as parts ' $a$ ' through ' $h$ ' of figure 3. In this figure, ' $a$ ' is the calculated lineshape for a paramagnetic site with an axial $g$-tensor and no hyperfine interaction (the simplest anisotropic case), and ' $b$ ' represents the field-derivative of the absorption in ' $a$ '. The absorption lineshape for an axial $g$-tensor which is perturbed by an isotropic hyperfine interaction and its corresponding derivative shape are given in ' $c$ ' and ' $d$ ', respectively. In ' $e$ ', the typical EPR absorption for a site exhibiting an orthorhombic $g$-tensor is shown, while in ' $f$ ' its derivative shape is recorded. ' $g$ ' represents the absorption lineshape corresponding to an orthorhombic $g$-tensor as in ' $e$ ', but exhibiting further isotropic hyperfine interaction; the corresponding derivative lineshape is depicted in ' $h$ '. In all these lineshapes, the $g$-values are determinable from the field positions indicated in the diagrarns, using $g^{\prime}=h v / \beta_{e} H$.

One simplifying assumption that has been implicitly made in the EPR resonance conditions, (1), (3) and (4), is that all tensor quantities have identical principal axes. This restriction can, of course, be removed by introducing additional adjustable trigono- 


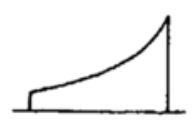

(a)

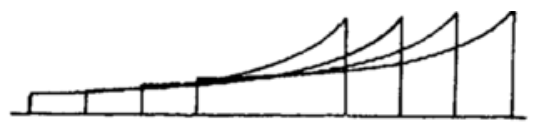

(c)

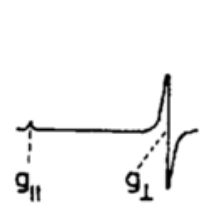

(b)

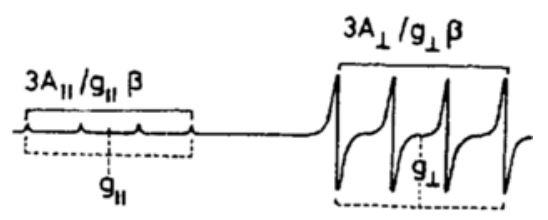

(d)
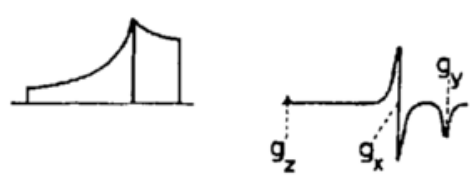

(e)

(f)

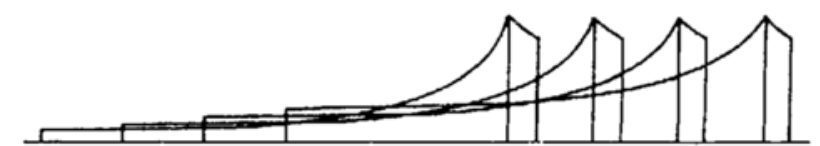

(g)
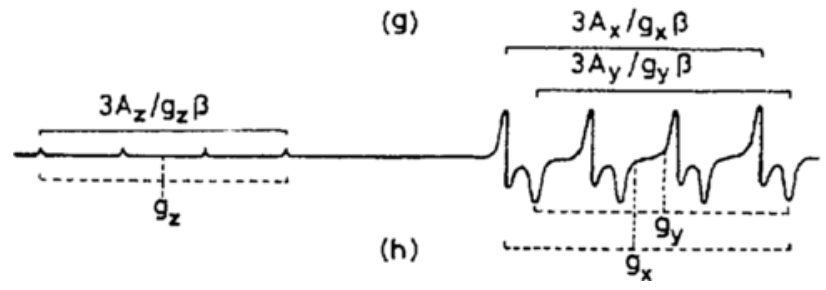

Figure 3. Typical EPR powder patterns computed for axial and non-axial g-tensor anisotropy. Parts (a)-(h) of the figure are explained in the text.

metric parameters to take account of the relative orientations of the various tensor axes. In specific instances, when the experimental lineshapes appear well-resolved, the extent of non-coincidence of the tensor axes may be reliably computed by trial-and-error lineshape simulation (Raghunathan and Sur 1984).

Powder NMR lineshape singularities similar to the EPR examples discussed above are known for quadrupolar solids. From the angular terms in (6), we see that the powder pattern will have two maxima corresponding to $\cos \theta=0$ and $\cos ^{2} \theta=5 / 9$. The frequencies where these maxima occur are given by the resonance conditions

$$
\begin{aligned}
& v^{I}\left(+\frac{1}{2} \leftrightarrow-\frac{1}{2}\right)=v_{0}+\left[v_{Q}^{2} / 16 v_{0}\right]\left[I(I+1)-\frac{3}{4}\right], \\
& v^{I I}\left(+\frac{1}{2} \leftrightarrow-\frac{1}{2}\right)=v_{0}-\left[v_{Q}^{2} / 9 v_{0}\right]\left[I(I+1)-\frac{3}{4}\right],
\end{aligned}
$$

leading to the central line splitting

$$
\Delta v=v^{I}-v^{I I}=\left[25 v_{Q}^{2} / 144 v_{0}\right]\left[I(I+1)-\frac{3}{4}\right] .
$$

Typical NMR powder patterns for the central transition in an $I=3 / 2$ system in the presence of second-order quadrupolar interaction (13) are shown in figure 4 for several 


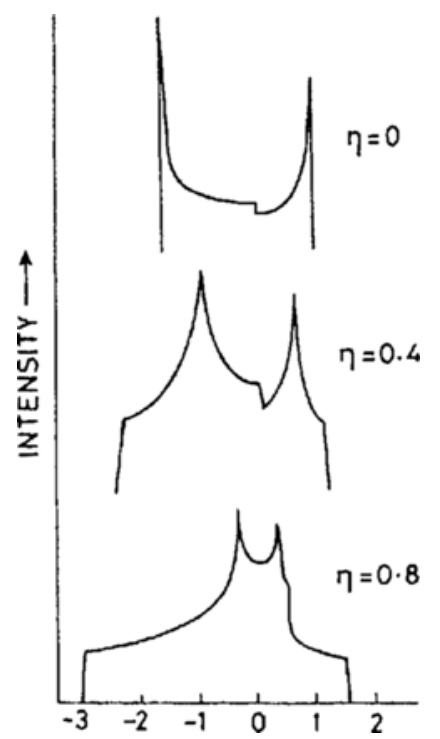

Figure 4. Typical NMR powder patterns for the central $\left(m_{1}=-\frac{1}{2} \leftrightarrow \frac{1}{2}\right)$ transition of $I=3 / 2$ nuclei in the presence of second-order quadrupolar interaction. Lineshapes computed for different $\eta$ values are shown. The horizontal axis is in units of $[I(I+1)-3 / 4] v_{Q}^{2} / 16 v_{0}$.

values of $\eta$. In a later section, the usefulness of these lineshapes in unravelling the types of boron coordination in borate glasses is demonstrated.

In the absence of axial symmetry for the EFG, $\eta \neq 0$, and (6) is replaced by a more complicated expression involving both $\theta$ and $\phi$ (Taylor et al 1975). Such expressions have been utilised, for example, in the computer-evaluated powder pattern of ${ }^{93} \mathrm{Nb}$ NMR in powdered sodium niobate (Wolf et al 1970).

\section{Lineshapes of amorphous materials: the need for 'random structure' models}

Magnetic resonance absorption in amorphous materials is often more complicated than in crystalline powders because here we have, in addition to a distribution of 'crystallite' orientations, an inherent distribution of the spin Hamiltonian parameters. Recent reviews (Griscom 1980; Rao and Rao 1985) of the EPR of transition metal ion sites in glasses document hosts of examples where such distribution models have been invoked. In such distributed systems, the overall population is expected to be represented by a probability density function, and this basic idea has been incorporated in various revent publications.

One of the earliest, and perhaps most extreme, cases of such distributed or 'strained' spin sites was reported in some high-melting metals, where the difficulty of annealing was the cause of the strain (McCart and Barnes 1968). This had the effect of 'washing out', or causing enormous line broadening effects in the NMR quadrupolar satellite positions of ${ }^{45} \mathrm{Sc},{ }^{9} \mathrm{Be}$ etc. The results of this work were explained by using a simple model of random electric field gradient inhomogeneity in the usual first order treatment of quadrupolar satellites. 
The idea of distributed Hamiltonian parameters has been incorporated to varying degrees in the analysis of magnetic resonance lines of vitreous inorganic and organic glasses as well. In an early investigation, Silver and Bray (1958) explained the broadening in the ${ }^{11} \mathrm{~B}$ NMR line of sodium-boron oxide glasses by a 'variation in the coupling constants'. Later, Kriz and Bray (1971), in their ${ }^{11} \mathbf{B}$ NMR study of glassy $\mathbf{B}_{2} \mathrm{O}_{3}$, reported a distribution in the boron sites which gave rise to a distribution in the quadrupole coupling constants. In a more general analysis one could, of course, take the view that random distribution exists both in the asymmetry parameters and quadrupole coupling constants.

In a very recent review, Bray (1985) has surveyed the relationship between glass structure and quadrupole or chemical shift effects in the NMR of various quadrupolar nuclei. The diagnostic guidelines provided in this survey ought to be quite useful in studies of the vitreous, glassy or amorphous quadrupolar solids. For example, the spectra to be expected for ${ }^{11} \mathrm{~B}$ NMR in borate glasses may be summarised as shown in figure 5. ${ }^{11} \mathrm{~B}$ in tetrahedrally bonded sites $\left(\mathrm{BO}_{4}\right.$ units) will exhibit little or no secondorder quadrupolar effects because of vanishingly small $\mathrm{EFG}$ components, and the result is a narrow, symmetric derivative line for the $m_{1}=\frac{1}{2} \rightarrow-\frac{1}{2}$ transition. Symmetric $\mathrm{BO}_{3}$ units (i.e. all bridging or all non-bridging oxygens) yield the response identified as

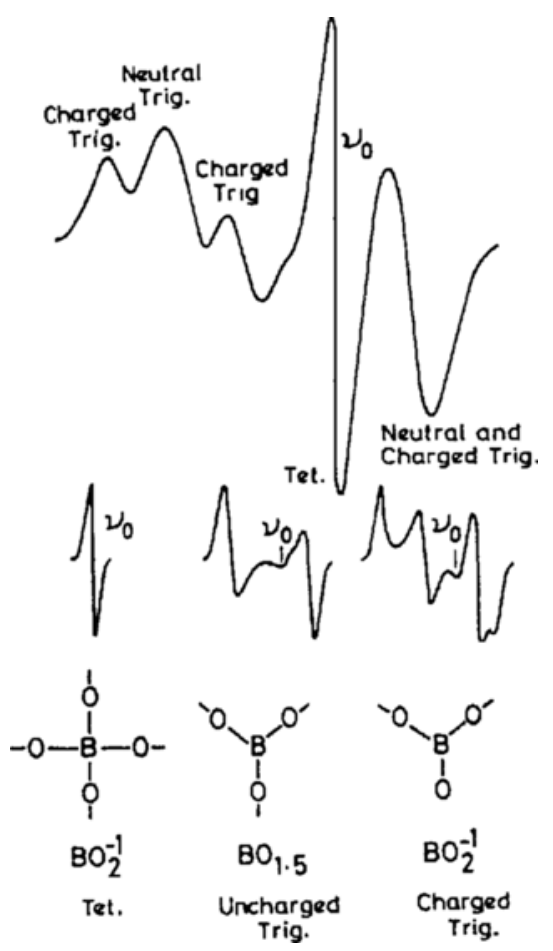

Figure 5. Computer simulation of a typical ${ }^{11} \mathrm{~B}$ NMR derivative spectrum in a borate glass is shown at the top. In the middle part of the figure, the upper spectrum is decomposed into shapes corresponding to charged trigonal, neutral trigonal and tetrahedral spin sites, which are shown in the bottom portion (after Bray 1985). 
'neutral trig' in figure 5. $\mathrm{BO}_{3}$ units with one or two non-bridging oxygens will have a non-zero $\eta$ value and will yield a spectrum marked as 'charged trig' in figure 5 (which is identifiable as one of the model lineshapes we have calculated earlier in figure 4).

In another interesting early contribution, Peterson and Kurkjian (1972) interpreted the frequency- (or field-) dependent broadenings of hyperfine structures in $\mathrm{Ti}^{3+}$ containing glasses by analysing the EPR lineshapes in terms of 'random glass structures', with variances (in the statistical sense) in $\overrightarrow{\mathbf{g}}$ and $\vec{A}$. Imagawa (1968), by considering a distribution in the single parameter, $g_{\|}$, was able to obtain semiquantitative fits of the EPR spectra of $\mathrm{Cu}^{2+}$ in alkali borate glasses. Later on, Kawazoe et al (1980) have also incorporated the distribution in $g_{\|}$and $A_{\|}$in their simulation of the EPR of $\mathrm{Cu}^{2+}$ sites in silicate, borate and phosphate glasses. The authors correlate large variances in $g_{\|}$with the coexistence of various borate groups 'competitively coordinating' to $\mathrm{Cu}^{2+}$. By comparing the much better-resolved lineshapes of $\left[\mathrm{Cu}(\mathrm{OH})_{4}\right]^{2-}$ in DMso-water 'glass' to the broad lines of $\mathrm{Cu}^{2+}$ in a $\mathrm{PbO}-\mathrm{B}_{2} \mathrm{O}_{3}$ glass, they conclude that the organic glass has a rigid structure maintained by hydrogen-bonding resulting in an almost identical environment for all $\mathrm{Cu}^{2+}$ sites, whereas the much decreased 'rigidity' of the lead oxide glass leads to a wide distribution of $\mathrm{Cu}^{2+}$ sites.

The distribution of zero field parameters in $\mathrm{Mn}^{2+}$-containing oxide glasses have been discussed by Kliava and Purāns (1980) and by Kliava (1982). Along somewhat analogous lines, Murai et al (1985) have emphasised the necessity of envisaging a $\overrightarrow{\mathrm{D}}$-tensor distribution in the EPR lineshape simulation of paraquinones in organic glassy matrices. Recently Hagen et al (1985b) have shown that paramagnetic sites in amorphous metalloenzymes have a ' $g$-strain' (i.e., distributed $g$-values) and have formulated a statistical method to achieve the simulation of the corresponding EPR lineshapes. In an accompanying paper, the authors (Hagen et al 1985a) give a numerical procedure which is claimed to be fast in simulating the lineshapes.

The large number of results surveyed are seen to fit into a broad classification called 'random structure models' since, in almost all of the cases cited, a probability density function has to be used to get the distribution of spin Hamiltonian parameters. The density function assumed is most likely to be a normal (i.e., Gaussian) distribution, which may be univariate or multivariate with or without correlation. We note that, in the case of multivariate normal distribution, a zero correlation implies statistical independence of $n$ variables and can be written as a product of $n$ univariate normal distributions.

Lineshape simulations based on the distributed spin Hamiltonian parameters can be broadly classified into two categories. In the first category we find the use of both the shape function and a probability density function. Here the probability density function is used just as a weight function, and the shape function is integrated as in a simple powder case (12). The other category, proposed by Peterson et al(1974a), defines the magnetic resonance line as the probability density function in the magnetic field variable. This method is devoid of the use of shape function and subsequent convolution.

In Peterson's procedure, the collection of all the paramagnetic species, each with an individual geometry $\omega_{i}$, is denoted by $\Omega$. For a simple ' $g$-only' case with axial symmetry, each of these $\omega_{i}$ can be mapped on to Euclidean three-space by a random vector $\mathbf{X}$ whose components are $g_{i,} g_{\perp}$ and $\theta, \theta$ is the angle between the magnetic field direction and the principal symmetry axis. The components of $\mathbf{X}$ can be described by a joint probability density function, $p\left(g_{\|,}, g_{\perp}, \theta\right)$. Since $\theta$ is statistically independent of $g_{\|}$and 
$g_{\perp}$, we have

$$
p\left(g_{\|}, g_{\perp}, \theta\right)=p\left(g_{\|}, g_{\perp}\right) \cdot p(\theta) .
$$

$p(\theta)$ is the well known function $\sin \theta$ in the domain $\theta=0$ to $\pi / 2$. $p\left(g_{\|,}, g_{\perp}\right)$ is assumed to be a bivariate normal density function with $\sigma_{\|}^{2}, \sigma_{\perp}^{2}$ as the variances and $\rho$ as the correlation coefficient between $g_{\|}$and $g_{\text {. }}$. The objective is to find out the probability density function of the magnetic field from the above given densities, such that the following resonance condition is satisfied

$$
h v=g \beta_{e} H=\left(g_{\|}^{2} \cos ^{2} \theta+g_{\perp}^{2} \sin ^{2} \theta\right)^{1 / 2} \beta_{e} H .
$$

This is achieved as follows. First the random vector $\mathbf{X}$ is transformed into a new random vector $\mathbf{Y}$ whose components are $g_{\mid j}, g_{\perp}$ and $g$. The new vector is described by the density function

$$
p\left(g_{i \mid}, g_{\perp}, g\right)=p\left(g_{\|}, g_{\perp}\right) \cdot p(\theta)|J|,
$$

where $|J|$ is the absolute value of the Jacobian associated with the transformation. For example, a small volume element $\mathrm{d} V_{a}$ in the first coordinate system may transform into two other volume elements $\mathrm{d} V_{b 1}$ and $\mathrm{d} V_{b 2}$ in the new coordinate system as shown in figure 6. After the transformation,

$$
p\left(g_{\|}, g_{\perp}, g\right)=\frac{g p\left(g_{\|}, g_{\perp}\right)}{\left[\left(g_{\|}^{2}-g_{\perp}^{2}\right)\left(g^{2}-g_{\perp}^{2}\right)\right]^{1 / 2}} .
$$

Now the probability density of $g$ is given by

$$
p(g)=\iint p\left(g_{\|}, g_{\perp}, g\right) \mathrm{d} g_{\|} \mathrm{d} g_{\perp} .
$$

The domain of this integration has to be carefully fixed because it depends on how $\mathbf{X}$ has been transformed into Y. Peterson et al (1974a) suggest the evaluation of these integrals by numerical methods, e.g., Simpson's $1 / 3$ rule with Richardson's extrapolation (see, Dahlquist and Björek 1974). In the integration, one has to avoid the singularities at $g_{\|}=g_{\perp}$ and $g_{\perp}=g$.

The final step in the above computation is to convert the density $g$ into that of

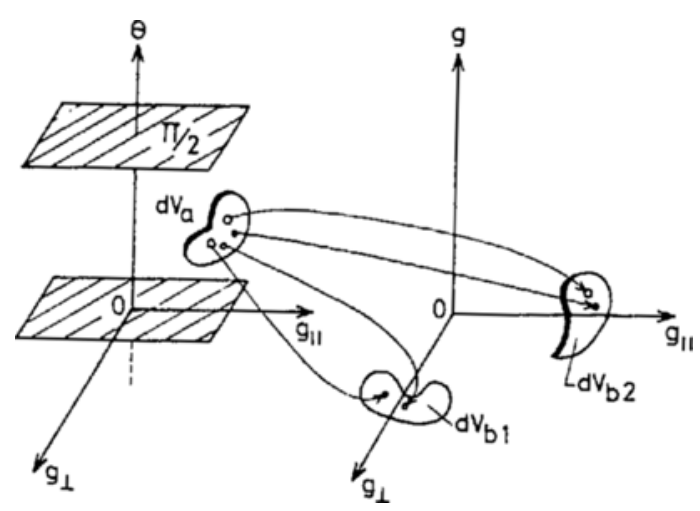

Figure 6. Coordinate transformation of domain $\mathrm{d} V_{a}$ into domains $\mathrm{d} V_{b 1}$ and $\mathrm{d} V_{b 2}$. 
magnetic field $H$ as given by

$$
p(H)=p(g) \cdot\left|\frac{h v}{\beta_{e} H^{2}}\right|,
$$

where, once again, $h v / \beta_{e} H^{2}$ is the Jacobian associated with the transformation. The consequences of the $g$ to $H$ transformation have been well documented (Peterson et al 1976; Carnevale et al 1976). For instance, a simple re-labelling of the axis to convert the $g$-density into $\mathrm{H}$-density may be erroneous. The last-mentioned transformation is essential for explaining the spectra recorded at different frequencies.

We have recently developed a new formalism in which all the three above-mentioned steps [viz., (i) derivation of the Jacobian and transformation of the random vector into another domain; (ii) integration of the transformed joint density to get the density of $g$, and (iii) transformation of the $g$ density to $H$ density] are automatically realised, since our computations are based on a more direct approach described in standard textbooks (see Papoulis 1965). In this approach, given the overall joint density of the random variables $x_{1}, x_{2}, \ldots x_{n}$ and the relation between these and a new variable $z$ such as, say, $z=h\left(x_{1}, x_{2}, \ldots x_{n}\right)$, the density of $z$, namely, $f(z)$, is given by

$$
f(z)=\frac{1}{\mathrm{~d} z} \iint_{\Delta D_{z}} \ldots \int f\left(x_{1}, x_{2}, \ldots x_{n}\right) \mathrm{d} x_{1} \mathrm{~d} x_{2}, \ldots \mathrm{d} x_{n} .
$$

In (20), the domain of the integration, $\Delta D_{2}$, is defined by the inequality

$$
z<h\left(x_{1}, x_{2}, \ldots x_{n}\right) \leqslant z+\mathrm{d} z .
$$

Using (20), the density of $H$ can be written as

$$
p(H)=\frac{1}{\mathrm{~d} H} \iiint_{\Delta 0_{H}} p\left(g_{\|}, g_{\perp}\right) \cdot p(\theta) \mathrm{d} g_{\|} \mathrm{d} g_{\perp} \mathrm{d} \theta,
$$

where $\Delta D_{H}$ is our domain of interest described by

$$
H<h v /\left[\left(g_{\|}^{2} \cos ^{2} \theta+g_{\perp}^{2} \sin ^{2} \theta\right)^{1 / 2} \beta_{e}\right] \leqslant H+\mathrm{d} H .
$$

Equation (22) is seen to be a triple-integral. The domain will have to be fixed by imposing our 'resonance condition' according to (23) as the upper and lower limits on one of the integrals. We find it easier to solve for $\theta$ given $g_{\|}, g_{\perp}$ and $H$ (or $H+\mathrm{d} H$ ). A pictorial representation of the 'domain' is given in figure 7. In this figure, for simplicity the domain involved in the evaluation of the density $p(g)$ from the densities, $p\left(g_{\|}, g_{\perp}\right)$ and $p(\theta)$ may be considered. In analogy with (20), the appropriate expression is given by

$$
p(g)=\frac{1}{\mathrm{~d} g} \iiint_{\Delta D_{g}} p\left(g_{\| ;}, g_{\perp}\right) p(\theta) \mathrm{d} g_{\|} \mathrm{d} g_{\perp} \mathrm{d} \theta,
$$

with the domain $\Delta D_{\theta}$ now being defined as

$$
g<\left(g_{\|}^{2} \cos ^{2} \theta+g_{\perp}^{2} \sin ^{2} \theta\right)^{1 / 2} \leqslant g+\mathrm{d} g .
$$

The 'domain-fixing' shall now be done as follows. Referring to the concentric circles around the $\theta$-axis at $\theta=\pi / 4$ in figure 7 , all the points lying outside the inner circle of 


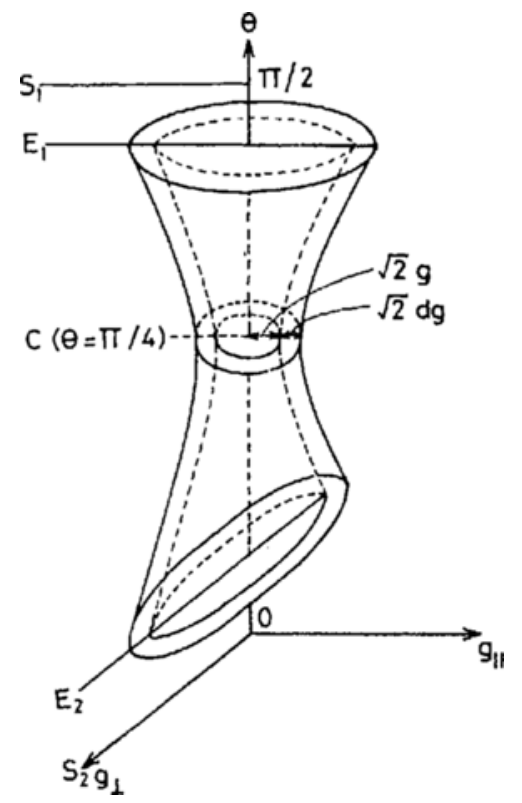

Figure 7. Pictorial representation of the integration domain $\Delta D_{\mathrm{a}}$ defined by (25) in the text. The labels $E_{1}, E_{2}, S_{1}$ and $S_{2}$ are discussed in the text. $C$ labels the plane of the concentric circles at $\theta=\pi / 4$

radius $\sqrt{2} g$ are seen to obey the inequality

$$
g<\left(g_{\|}^{2} \cos ^{2} \theta+g_{\perp}^{2} \sin ^{2} \theta\right)^{1 / 2} .
$$

At the same $\theta=\pi / 4$ value, all the points lying inside and on the circumference of the circle of radius $\sqrt{2}(g+\mathrm{d} g)$ are described by the inequality:

$$
\left(g_{\|}^{2} \cos ^{2} \theta+g_{\perp}^{2} \sin ^{2} \theta\right)^{1 / 2} \leqslant g+\mathrm{d} g .
$$

We therefore see that the domain $\Delta D_{g}$ at $\theta=\pi / 4$ is the area enclosed between the two concentric circles aforementioned. Between $\theta=\pi / 4$ to $\theta=\pi / 2$, figure 7 shows that the domain may be described by the area between two concentric ellipses $\left(E_{1}\right)$ with a common major axis parallel to the $g_{\|}$axis. Exactly at $\theta=\pi / 2$, the concentric ellipses become two strips $\left(S_{1}\right)$ running between $-\infty$ and $+\infty$ in the $g_{\|}$direction. The breadths of the inner and outer strips are, respectively, $2 g$ and $2(g+\mathrm{d} g)$ along $g_{\perp}$, with the $\theta$-axis passing throuth the centre of the strips. In the same way, we can see that between $\theta=\pi / 4$ and $\theta=0$ the domain $\Delta D_{g}$ is described by concentric ellipses $E_{2}$ and the strips at $S_{2}$. But now, the strips and the (common) major axis of the ellipses are parallel to the $g_{\perp}$ axis. The points $\theta=\pi / 2$ and $\theta=0$ represent the 'singularities'.

Although a pictorial visualisation of the equivalent for $p(H)$ may be difficult, we can nevertheless write the integral as

$$
p(H)=\frac{1}{\mathrm{~d} H} \int_{\theta_{1}}^{\theta_{2}} \int_{\theta_{3}}^{\theta_{4}} p\left(g_{\|}, g_{\perp}\right) \mathrm{d} g_{\|} \mathrm{d} g_{\perp} \int_{\theta_{1}}^{\theta_{2}} \sin \theta \mathrm{d} \theta .
$$


In (28) the limits for $g_{\|}$and $g_{\perp}$ are fixed by truncation of $p\left(g_{\|}, g_{\perp}\right)$ such that beyond the truncated limits $\left(g_{1}, g_{2}\right.$ for $g_{\|}$and $g_{3}, g_{4}$ for $\left.g_{\perp}\right)$ the density is negligible. After truncation, we normalize the density and carefully define the density only in the positive quadrant; that is, $g_{\|}$and $g_{\perp}$ are always given as positive non-zero numbers. The upper and lower limits of $\theta$ where the resonance condition is imposed are given as

and

$$
\theta_{1}=\sin ^{-1}\left\{\left[\left(h v / \beta_{e} H\right)^{2}-g_{\|}^{2}\right] /\left(g_{\perp}^{2}-g_{\|}^{2}\right)\right\}^{1 / 2}
$$

$$
\theta_{2}=\sin ^{-1}\left\{\left[\left(h v / \beta_{e}(H+\mathrm{d} H)\right)^{2}-g_{\|}^{2}\right] /\left(g_{\perp}^{2}-g_{\|}^{2}\right)\right\}^{1 / 2} .
$$

We employ a Monte Carlo integration technique based on systematic sampling (Kahn 1956) to estimate the outer double integral in (28). The inner integral is the integrand for the outer double integral, for which the simple analytical solution $(-\cos \theta)$ is available. This procedure cuts down the computer time by at least one order of magnitude. We stress that our formalism is applicable with equal facility to cases where more distributed parameters have to be included (e.g., hyperfine, zero field splittings etc.). We have been able to reproduce several of the results appearing in the literature using our new formalism. A complete lineshape simulation for an experimental glass spectrum exhibiting hyperfine splittings is currently in progress.

The methodology we have described above is also useful for simulating the NMR lines by making obvious changes in the variables. In the simplest case where $\eta=0$, either the quadrupolar coupling or the Larmor frequency, or both together, can become distributed (Peterson et al 1974b). Non-zero asymmetry parameters have also been considered in other simulations (Peterson et al 1975).

In one specific application of the density-function method, Peterson et al (1974a) have been able to generate the $g=4.3$ resonance line in $\mathrm{Fe}^{3+}$-containing glasses by considering the mean values 2.0 and 6.0 , respectively, for $g_{\|}$and $g_{\perp}$ and a large variance with negative correlation coefficient. This particular example, where a distributed single site has been predicted, is questioned by Momo et al (1981). The authors consider the constancy of the experimental line position with respect to temperature change as evidence against the statistically distributed model. However, it should be noted that the line position is mainly dependent on the mean values, whereas the width of the line is determined by variances. However, in a related paper Brodbeck (1980) concludes that in $\mathrm{Fe}^{3+}$ the fully rhombic crystal field limit uniquely generates the negative $g$-correlation required for spiking at $g=4 \cdot 3$.

Considerable caution has to be exercised in the interpretation of probability density parameters. For example, Kliava (1982) erroneously considers that, when the correlation coefficient $\rho= \pm 1$, the joint Gaussian density can be written as a product of two Gaussian densities. In fact, when $\rho= \pm 1$ the density is not well defined and has a singularity. Again, Brodbeck (1980), instead of using the complete density function description of $g_{\|}$and $g_{\perp}$, simply uses the linear relationship $\left(g_{\|}-2\right) /\left(g_{\perp}-6\right)=-1$ and then uses a shape function to fit the experimental EPR curves.

\section{Conclusion}

Over the years magnetic resonance lineshape simulation procedures have become very well-established diagnostic aids for the analysis of powder spectra. In favourable examples where the randomly oriented paramagnetic sites have only a minor spread in 
their resonance parameters, present lineshape simulation procedures could often faithfully reproduce all the experimental shapes. In the amorphous or 'glassy' state, however, it often becomes necessary to view the measurable spin resonance parameters as being distributed according to a probability density function. The present probability-theory based approaches we have discussed ought to lead to reliable estimates of the statistical parameters of the distribution (such as variances and correlation coefficients). This should result in an enhanced understanding of the amorphous state of matter.

\section{References}

Bleaney B 1960 Proc. Phys. Soc. (London) 75621

Bloch F 1946 Phys. Rev. 70460

Bray P J 1985 J. Non-Cryst. Solids 7319

Brodbeck C M 1980 J. Non-Cryst. Solids 40305

Carnevale A, Peterson G E and Kurkjian C R 1976 J. Non-Cryst. Solids 22269

Cohen M H and Reif F 1957 Solid state physics (eds) F Seitz and D Turnbull (New York: Academic Press) vol. 5

Coope J A R 1969 Chem. Phys. Lett. 3589

Dahlquist $\mathrm{G}$ and Bjōrck A 1974 Numerical methods (New Jersey: Prentice-Hall) chap. 7

Griscom D L 1980 J. Non-Cryst. Solids 40211

Hagen W R, Hearshen D O, Harding L J and Dunham W R 1985a J. Magn. Reson 61233

Hagen W R, Hearshen D O, Sands R H and Dunham W R 1985b J. Magn. Reson. 61220

Imagawa H 1968 Phys. Status Solidi 30469

Isomoto A, Watari H and Kotani M 1970 J. Phys. Sac. Jpn 291571

Jinguji M, Lin K C, McDowell C A and Raghunathan P 1976 J. Chem. Phys. 653910

Jones W H Jr, Graham T P and Barnes R G 1963 Phys. Rev. 1321898

Kahn H 1956 in Symposium on Monte Carlo methods (ed.) H A Meyer (New York: John Wiley)

Kawazoe H, Hosona H, Kokumai H, Nishii J and Kanazawa T 1980 J. Non-Cryst. Solids 40291

Kliava J 1982 J. Phys. C15 7017

Kliava J and Purafis J 1980 J. Magn. Reson. 4033

Kottis P and Lefebvre R 1964 J. Chem. Phys. 41379

Kriz H and Bray P J 1971 J. Non-Cryst. Solids 627

Lee $\mathrm{S} 1981$ Phys. Rev. $\mathrm{B23} 6151$

Lee S, Brown I M and Ames D P 1985 J. Chem. Phys. 831551

Maruani J, McDowell C A, Nakajima H and Raghunathan P 1968 Mol. Phys. 14349

McCart B R and Barnes R G 1968 J. Chem. Phys. 48127

McDowell C A, Raghunathan P and Tait J C 1973 J. Chem. Phys. 595858

Momo F, Ranieri G A and Sotgiu A 1981 J. Non-Cryst. Solids 46115

Müller-Warmuth W and Eckert H 1982 Phys. Rep. 8891

Murai H, Minami M, Hayakshi T and rHaya Y J 1985 Chem. Phys. 9333

Papoulis A 1965 Probability, random variables and stochastic processes (New York: McGraw-Hill) p. 235 Peterson G E and Kurkjian C R 1972 Solid State Commun. 111105

Peterson G E, Kurkjian C R and Carnevale A 1974a Phys. Chem. Glasses 1552

Peterson G E, Kurkjian C R and Carnevale A 1974b Phys. Chem. Glasses 1559

Peterson G E, Kurkjian C R and Carnevale A 1975 Phys. Chem. Glasses 1663

Peterson G E, Kurkjian C R and Carnevale A 1976 Phys. Chem. Glasses 1788

Raghunathan P 1981 Proc. Indian Acad. Sci. (Chem. Sci.) 90467

Raghunathan P 1982 in Solid state spectroscopy (ed.) S. Radhakrishna (Madras: COSTED International

Workshop Publications) chap. 18

Raghunathan P and Sur S K 1984 J. Am. Chem. Soc. 1068014

Rao K J and Rao B G 1985 Proc. Indian Acad. Sci. (Chem. Sci) 95169

Silver A H and Bray P J 1958 J. Chem. Phys. 29984

Taylor P C, Baugher J F and Kriz H M 1975 Chem. Rev. 75203 
Taylor P C, Ware D A and Bray P J 1968 J. Chem. Phys. 485158 Van Vleck J H 1948 Phys. Rev. 741168

Weil J A and Hecht H G $1963 \mathrm{~J}$, Chem. Phys. 38281

Wolf F, Kline D and Story H S 1970 J. Chem. Phys. 533538 